\title{
Aplicação de processo fotocatalítico mediado por dióxido de titânio para degradação de Sertralina
}

\author{
Ailton Jose Moreira ${ }^{a}$ Caroline Piza Maldib*, Gian Paulo Giovanni Freschia \\ a Programa de Pós-Graduação em Química, Universidade Federal de Alfenas, Poços de Caldas, 37715-400, Minas Gerais, Brasil. \\ b Laboratório de Fotólise, Fotocatálise e Especiação Química, Universidade Federal de Alfenas, Poços de Caldas, 37715-400, Minas \\ Gerais, Brasil. *carolinepizamaldi@gmail.com
}

Recebido: 23 agosto 2018 / Aceito: 11 outubro 2018 / Publicado online: 2 janeiro 2019

\begin{abstract}
Resumo
Esta pesquisa avaliou o potencial de degradação do fármaco Sertralina por meio de processos de fotocatálise, com a combinação de energia ultravioleta (UV) e diferentes semicondutores. Ensaios fotocatalíticos foram realizados em $10 \mathrm{~mL}$ de soluções de Sertralina com concentrações $10 \mathrm{mgL}^{-1}$, adicionados dos diferentes semicondutores $(10 \mathrm{mg})$. As amostras foram submetidas a radiação (UV) em cinco tempos de degradação $(0,5,10,30,60$ e 120 minutos). A determinação da concentração da Sertralina foi por cromatografia líquida de alta eficiência com deteç̧ão UV (CLAE-UV). Degradações de Sertralina de 51\%, 74\%, 75\% e $83 \%$ foram determinadas após aplicação da fotólise, fotocatalise $\mathrm{TiO}_{2}, \mathrm{TiO}_{2}-\mathrm{Mn}_{\mathrm{N}} \mathrm{TiO}_{2}-\mathrm{B}$, respectivamente. Assim, a maior eficiência no processo de degradação, foi determinado para o semicondutor de $\mathrm{TiO}_{2}$ dopado com Boro. Deste modo, a aplicação do processo de fotocatálise pode ser de grande importância nos processos de tratamento de efluentes e, a inserção de agentes dopantes pode tornar os semicondutores mais ativos em processos de fotocatálise.
\end{abstract}

Palavras-chave: Ultravioleta, fármaco, semiconductor, contaminantes emergentes.

\section{Application of photocatalytic process mediated by titanium dioxide for degradation of Sertraline}

\begin{abstract}
This study evaluated the degradation potential of the drug Sertraline by means of photocatalysis processes, with the combination of ultraviolet (UV) energy and different semiconductors. Photocatalytic assays were performed in $10 \mathrm{~mL}$ of Sertraline solutions containing $10 \mathrm{mg} \mathrm{L}^{-1}$, added to the different semiconductors $(10 \mathrm{mg})$. The samples were submitted to radiation (UV) in five degradation times $(0,5,10,30,60$ and 120 minutes). The determination of Sertraline concentration was by high performance liquid chromatography with UV detection. (HPLC-UV). Sertraline degradations of $51 \%, 74 \%, 75 \%$ and $83 \%$ were determined after application of photolysis, photocatalysis $\mathrm{TiO}_{2}, \mathrm{TiO}_{2}-\mathrm{Mn}$ and $\mathrm{TiO}_{2}-\mathrm{B}$, respectively. Thus, the higher efficiency in the degradation process was determined for the boron-doped $\mathrm{TiO}_{2}$ semiconductor. Thus, the application of the photocatalysis process can be of great importance in the effluent treatment processes, and the insertion of doping agents can make semiconductors more active in photocatalysis processes.
\end{abstract}

Keywords: Ultraviolet, drug, semiconductor, emerging pollutants.

\section{Introdução}

Produtos químicos são amplamente utilizados na sociedade moderna, sendo produzidos mundialmente em larga escala para as mais variadas aplicações. No entanto, uma das desvantagens da produção e utilização destes está nos resíduos que são gerados, sejam eles derivados diretamente das atividades industriais ou produzidos após seu consumo pela sociedade, que podem impactar de forma negativa o meio ambiente (Silva \& Collins, 2011).

A presença de fármacos residuais no meio ambiente pode apresentar efeitos adversos em organismos aquáticos $\mathrm{e}$ terrestres. Este efeito pode ocorrer em qualquer nível da hierarquia biológica, tais como célula, órgão, organismo, população e ecossistema, e podem ser observados em concentrações na ordem de ng L-1 . (Suchara, 2007). Dentre eles, está o cloridrato de sertralina, que pertence à classe dos denominados inibidores seletivos da recaptação da serotonina (SSRIs), amplamente utilizado como antidepressivo.

Estudo realizado em um estuário na Flórida, contaminado pelo lançamento de efluentes, detectou em amostras de plasma de tubarões da espécie Carcharhinusleucas a presença de diversos fármacos de uso humano, dentre os quais: Citalopram, Fluoxetina, 
Fluvoxamina, Paroxetina, Sertralina, Venlafaxina, sugerindo a acumulação destas substâncias nestes animais. (Junior, Pletsch \& Torres, 2014).

Os processos convencionais de tratamento, a que são submetidos os esgotos domésticos, baseados na degradação biológica dos contaminantes, não são eficientes para a completa remoção de fármacos residuais por possuírem ação biocida ou estruturas químicas complexas não passíveis de biodegradação. (Melo, Trovó, Bautitz \& Nogueira, 2009). Assim, os processos oxidativos avançados (POA) têm sido extensivamente estudados para tratamento de uma variada classe de compostos orgânicos (Moreira, Borges \& Gouvea, 2017; Pliego et al., 2013; Cesaro \& Belgiorno, 2016), devido ao seu potencial como alternativas ou complementos aos processos convencionais de tratamento de efluentes.

Para os processos fotocatalíticos heterogêneos, a inserção de diferentes semicondutores pode ser aplicada, pois estes, ao atuarem como catalizadores, aumentam a eficiência do processo de degradação. Esta eficiência do semicondutor pode ser otimizada com a inserção de elementos dopantes, possibilitando alteração nas propriedades físicas do semicondutor, e assim, tornando-os mais fotoativos. (Santos, Oehninger, Willing \& Rosa, 2017; Cruz, Henning, Santos \& Peralta, 2010).

Essa pesquisa teve como objetivo avaliar o potencial de degradação do fármaco Sertralina por meio dos processos de fotocatálise, onde a aplicação de diferentes semicondutores, baseados em $\mathrm{TiO}_{2}$, são utilizados como potenciais catalisadores no processo de degradação.

\section{Material e Métodos}

O método de síntese do semicondutor de $\mathrm{TiO}_{2}$ (Peng, Loa, Oua \& Lai, 2010), consistiu em dispersar os 12,6 mL de tetraisopropóxido de titânio $\left(\mathrm{C}_{12} \mathrm{H}_{28} \mathrm{O}_{4} \mathrm{Ti}\right)$ em $60 \mathrm{~mL}$ de solução de isopropanol $\left(\mathrm{C}_{3} \mathrm{H}_{7} \mathrm{OH}\right) 20 \%\left(\mathrm{v} \mathrm{v}^{-1}\right)$ em béquer de vidro de 250 $\mathrm{mL}$. A mistura foi agitada durante $1 \mathrm{~h}$ com agitação magnética, seguida do aquecimento no micro-ondas (CEM, Matthews, NC, EUA), aplicando o seguinte programa de aquecimento: Etapa 1$40^{\circ} \mathrm{C}$, de 0 a 20 min; Etapa $2-60{ }^{\circ} \mathrm{C}$ de 21 a 40 min e Etapa 3 - $200^{\circ} \mathrm{C}$ de 41 a $60 \mathrm{~min}$. Após o aquecimento, as amostras foram resfriadas e submetidas a centrifugação por 10 min à $3200 \mathrm{rpm}$. Este procedimento é realizado 3 vezes, o precipitado é lavado com metanol e recolhido em béquer de vidro de $200 \mathrm{~mL}$ a cada lavagem. Subsequentemente, as amostras são submetidas à primeira calcinação a $200{ }^{\circ} \mathrm{C}$ por $1 \mathrm{~h}$, em um forno de mufla FI 1200 (CEVIPLA, São Carlos, SP, Brasil), com a rampas de aquecimento de: $27^{\circ} \mathrm{C}$ a $200{ }^{\circ} \mathrm{C}$ com uma taxa de aquecimento de $5{ }^{\circ} \mathrm{C} \mathrm{min}^{-1}$, permanência a $200{ }^{\circ} \mathrm{C}$ por $1 \mathrm{~h}$, e resfriamento de $200{ }^{\circ} \mathrm{C}$ a $27^{\circ} \mathrm{C}$ com taxa de resfriamento de $5{ }^{\circ} \mathrm{C} \mathrm{min}^{-1}$. Após a primeira calcinação, as amostras foram maceradas em almofariz de ágata, para melhor homogeneização das partículas, transferidas para barca de porcelana e submetidas a uma segunda calcinação de $600{ }^{\circ} \mathrm{C}$, com a rampa de aquecimento de: $27^{\circ} \mathrm{C}$ a $600{ }^{\circ} \mathrm{C}$ com taxa de aquecimento de $5{ }^{\circ} \mathrm{C} \mathrm{min}^{-1}$, permanecendo a $600{ }^{\circ} \mathrm{C}$ por $2 \mathrm{~h}$ e resfriamento de $600{ }^{\circ} \mathrm{C}$ a $27^{\circ} \mathrm{C}$ com taxa de resfriamento de $5^{\circ} \mathrm{C} \mathrm{min}^{-1}$.

$\mathrm{O}$ processo de síntese das amostras contendo o Mn e B como dopantes, foram idênticos ao processo descrito acima, sendo que acetato de manganês tetrahidratado $\left(\mathrm{Mn}\left(\mathrm{CH}_{3} \mathrm{COO}\right)_{2} \cdot 4 \mathrm{H}_{2} \mathrm{O}\right)$ e ácido bórico $\left(\mathrm{H}_{3} \mathrm{BO}_{3}\right)$ foram adicionados ao sistema após a adição das soluções de Isopropóxido de titânio e isopropanol, e anteriormente a etapa de aquecimento no micro-ondas. A adição foi executada através da transferência do reagente sólido ao sistema reacional, na quantidade em massa $(\mathrm{g})$, que representasse $1 \%(\mathrm{~m} / \mathrm{m})$ do dopante $(\mathrm{Mn}$ ou $\mathrm{B})$ em relação ao titânio. As amostras contendo os dopantes foram denominadas de $\mathrm{TiO}_{2} 1 \% \mathrm{Mn}$ e $\mathrm{TiO}_{2} 1 \% \mathrm{~B}$.

Usou-se o espectrofotômetro UV-Vis Cary 60, combinado com uma célula de quartzo de caminho óptico de $1 \mathrm{~cm}$ para determinar o comprimento de onda do método cromatográfico. $\mathrm{O}$ equipamento, com faixa de comprimento de onda de 190 a 1100 nm, com seleção manual e automática, foi empregado com velocidade de varredura de $24.000 \mathrm{~nm} \mathrm{~min}^{-1}$ e largura de banda espectral de $1,5 \mathrm{~nm}$.

Para execução dos ensaios de fotodegradação, $10 \mathrm{~mL}$ da amostra foram transferidos para béquer de vidro de $50 \mathrm{~mL}$, sendo inseridos no interior do fotoreator. Todas as amostras foram irradiadas sob agitação, para melhor dispersão dos semicondutores, quando for o caso, e também, melhor homogeneidade da solução de Sertralin, durante o processo de irradiação. $\mathrm{O}$ fotoreator $\mathrm{UV}$, aplicado nesta pesquisa possui as configurações descritas segundo Moreira, Pinheiro, Araújo \& Freschi, 2016.

As determinações cromatográficas foram realizadas empregando a cromatografia líquida de alta eficiência (HPLC, Modelo Agilent 1220 Infinity LC, Santa Clara, CA, EUA) equipado com um amostrador automático (G4290B), uma coluna com controle de temperatura, detector UV/Vis (faixa de leitura de 190 a 600nm) e uma coluna cromatográfica Zorbax Eclipse Plus C18. 4,6 x 250 $\mathrm{mm}, 5 \mu \mathrm{m}$.

O processamento dos dados cromatográficos foi realizado pelo software EZChrom do Sistema de Dados Cromatográficos (CDS) Agilent OpenLAB. As condições cromatográficas utilizadas foram: fluxo da fase móvel de 1 $\mathrm{mL} \min ^{-1}$, temperatura da fase móvel controlada em $35^{\circ} \mathrm{C}$, volume de injeção da amostra de $30 \mu \mathrm{L}$, comprimento de onda $205 \mathrm{~nm}$ e gradiente de eluição nas seguintes proporções:0 min a 2 min: $85 \%$ ACN e $15 \% \mathrm{PO}_{3}-\mathrm{pH}=3$, de 2 min a 4 min: $65 \%$ ACN e $35 \% \mathrm{PO}_{3}-\mathrm{pH}=3$, de $4 \mathrm{~min}$ a $10 \mathrm{~min}: 35 \% \mathrm{ACN}$ e $65 \% \mathrm{PO}_{3}-\mathrm{pH}=3$ e, $10 \mathrm{~min}: 10 \%$ $\mathrm{ACN}$ e $90 \% \mathrm{PO}_{3}-\mathrm{pH}=3$. Todas as amostras submetidas à irradiação foram analisadas no HPLC em triplicata.

A eficiência de cada processo de degradação foi calculada através dos dados de remoção percentual de Sertralina e suas respectivas constantes cinéticas de degradação, que foram determinadas para os tempos de irradiação aplicados.

A solução padrão (estoque) $1000 \mathrm{mgL}^{-1}$ de Sertralina (SRTL) $\left(\mathrm{C}_{17} \mathrm{H}_{17} \mathrm{C}_{12} \mathrm{~N}\right)$ foram preparadas por meio da dissolução do respectivo sal 98,49\% em Metanol grau HPLC e as soluções intermediárias foram preparadas a partir da diluição da solução estoque de Sertralina com água ultrapura, obtida por deionização em sistema Milli-Q Plus (Millipore, Bedford, MA, EUA), na concentração de 10 $\mathrm{mgL}^{-1}$ e $\mathrm{pH}=6,5$.

As soluções tampão de Fosfato Monobásico de Potássio 
$\left(\mathrm{KH}_{2} \mathrm{PO}_{4}\right) \mathrm{pH}=3,0$, são preparadas através da dissolução do respectivo sal (Sigma Aldrich, EUA) em água ultrapura, sendo o pH ajustado com Ácido Fosfórico $85 \%$ (Neon) $\left(\mathrm{H}_{3} \mathrm{PO}_{4}\right)$, para utilização nas análises cromatográficas da Sertralina.

Solventes grau HPLC, como Acetonitrila (ACN) $\left(\mathrm{C}_{2} \mathrm{H}_{3} \mathrm{~N}\right)$, Metanol (JT Backer), foram usados nos procedimentos para identificação e quantificação da Sertralina por cromatografia líquida de alta eficiência.

Solução de Ácido Nítrico $10 \%$ (v/v) $\left(\mathrm{HNO}_{3}\right)$, preparado através da diluição em água de Ácido Nítrico 65\%, aplicado na lavagem das vidrarias e materiais utilizados.

\section{Resultados e Discussão}

A Figura 1 reporta a degradação fotolítica e fotocatalítica da Sertraline, sendo possível constatar que, o decaimento da concentração do fármaco evidencia a degradação do mesmo após o processo de irradiação UV. A variação da concentração nos 3 primeiros minutos de fotodegradação foi calculada em 7 , 35,20 e $25 \%$, para os processos fotolíticos (UV) e fotocatalíticos $\mathrm{TiO}_{2}, \mathrm{TiO}_{2} 1 \% \mathrm{Mn}$ e $\mathrm{TiO}_{2} 1 \% \mathrm{~B}$, respectivamente. No meio ambiente, a Sertraline é identificada como sendo um fármaco de elevada persistência, uma vez que, para um período de hidrolise e fotólise de 8 h, sua remoção máxima foi de até 2 e 6\%, respectivamente, para os diferentes processos (Styrishave, Halling-Sorensen \& Ingerslev, 2011).

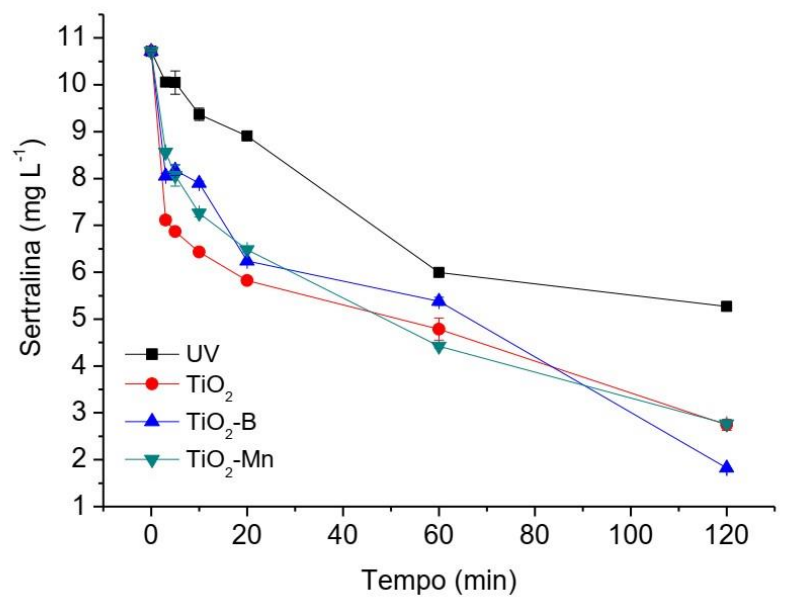

Figura 1. Degradação fotocatalítica UV da Sertraline.

Os resultados de fotodegradação confirmam que, a inserção dos semicondutores exerce influência positiva na degradação da SRTL, sendo que taxas de remoção da ordem de $51 \%$ (fotólise), $74 \%\left(\mathrm{TiO}_{2}\right), 75 \%\left(\mathrm{TiO}_{2} 1 \% \mathrm{Mn}\right)$ e $83 \%\left(\mathrm{TiO}_{2} 1 \% \mathrm{~B}\right)$ foram verificados para tempo de 120 minutos de irradiação (Figura 2). Obviamente que, durante a aplicação de processos fotocatalíticos, adsorção na superfície do semicondutor pode ser constatada (Chen et al., 2016; Kaplan, Erjavec, Drazi, Gradadolnikc, Pintar, 2016), porém, sendo a interação analito/semicondutor, essencial para que os processos fotocatalíticos sejam efetivos, conclui-se que as etapas de adsorção são importantes e desejáveis. Assim, a variação mais acentuada (de 20 a 35\%) da concentração inicial da Sertralina no tempo de até $3 \mathrm{~min}$, pode estar mais associada aos processos adsorção, do que propriamente a degradação fotocatalítica, que ocorre de modo mais significativo nos tempos de irradiação posteriores aos $3 \mathrm{~min}$.

A determinação de constante cinética de pseudo primeira ordem são aplicadas (Styrishave et al., 2011; Perini \& Nogueira, 2015), a fim de se avaliar melhor a eficiência da degradação da Sertralina. Através dos valores de constantes cinéticas obtidos para os diferentes processos de degradação (Figura 2), observa-se em comparação ao processo fotolítico, um aumento de $31 \%\left(\mathrm{TiO}_{2}\right), 60 \%$ $\left(\mathrm{TiO}_{2} 1 \% \mathrm{Mn}\right)$ e $107 \%\left(\mathrm{TiO}_{2} 1 \% \mathrm{~B}\right)$ para os processos fotocatalíticos. Importante destacar ainda que, para todos os processos, a correlação linear para o intervalo de tempo aplicado (5 a 120 minutos) apresenta valores confiáveis, correspondentes a $\mathrm{R}^{2}=0,902$ (Fotólise), $\mathrm{R}^{2}=0,984\left(\mathrm{TiO}_{2}\right)$, $\mathrm{R}^{2}=0,938\left(\mathrm{TiO}_{2} 1 \% \mathrm{~B}\right)$ e $\mathrm{R}^{2}=0,982\left(\mathrm{TiO}_{2} 1 \% \mathrm{Mn}\right)$.

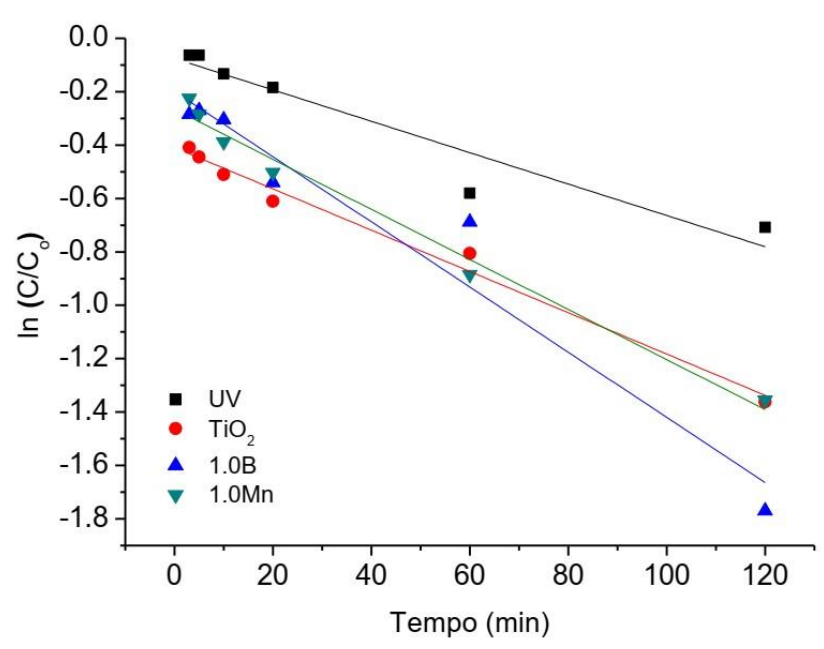

Figura 2. Cinética de pseudo primeira ordem obtido durante a degradação fotolítica e fotocatalítica da sertralina.

A constante cinética de degradação foi determinada em $\mathrm{k}=5,88 \times 10^{-3} \mathrm{~min}^{-1}$ (fotólise UV), $\mathrm{k}=7,73 \times 10^{-3} \mathrm{~min}^{-1}$ $\left(\mathrm{TiO}_{2}\right), \mathrm{k}=9,38 \times 10-3 \mathrm{~min}^{-1}\left(\mathrm{TiO}_{2} 1 \% \mathrm{Mn}\right)$ e $\mathrm{k}=12,2 \times 10-$ $3 \mathrm{~min}^{-1}\left(\mathrm{TiO}_{2} 1 \% \mathrm{~B}\right)$. Os valores de constante cinética obtidos para a fotocatálise representam um aumento de $31 \%\left(\mathrm{TiO}_{2}\right), 56 \%\left(\mathrm{TiO}_{2} 1 \% \mathrm{Mn}\right)$ e $107 \%\left(\mathrm{TiO}_{2} 1 \% \mathrm{~B}\right)$ quando comparados a fotólise, reafirmando que a inserção dos semicondutores contribui de modo positivo para a degradação da Sertralina. As cinéticas de degradação obtidas por Perini \& Nogueira (2015) foi $\mathrm{k}=2,2 \times 10^{-3} \mathrm{~min}^{-}$ 1 em $\mathrm{pH}=6,5$ (mesma condição de $\mathrm{pH}$ aplicada neste estudo), representando um valor $82 \%$ menor do que o obtido para o sistema fotocatalítico $\mathrm{TiO}_{2} 1 \% \mathrm{~B}$, que foi aplicado nesta pesquisa.

Nos estudos conduzidos por Perini \& Nogueira (2015), a concentração inicial de Sertralina apresentou valor de 22 $\mathrm{mg} \mathrm{L}^{-1}$, enquanto que para o presente estudo, a concentração aplicada foi de $10 \mathrm{mg} \mathrm{L}^{-1}$. Após otimização de parâmetros como o $\mathrm{pH}$, eles alcançaram cinética de degradação de $\mathrm{k}=1,4 \mathrm{~min}^{-1}$ (com adição de peróxido e $\mathrm{pH}$ $=2,5$ ), além outros valores que superam os verificados neste estudo, evidenciando que o controle de $\mathrm{pH}$ inicial da solução é essencial para obtenção de melhores taxas de degradação da Sertralina.

Estudos de degradação da Sertralina conduzidos por 
Shen et al. (2011) na presença de peróxido de hidrogênio, também obtive as menores taxas de degradação em $\mathrm{pH}$ mais básico, e deste modo, a degradação da Sertralina pode ser otimizada para valores de $\mathrm{pH}$ mais ácidos. Como não foi aplicado ajuste de $\mathrm{pH}$ no presente estudo, podemos sugerir que as taxas de remoção de Sertralina poderiam ter sido maiores com a otimização deste parâmetro, que deve ser avaliado para estudos futuros.

Os resultados obtidos neste estudo demonstram que, a Sertralina sofre degradação fotolítica sob radiação UV, mas que essa degradação é otimizada quando semicondutor é adicionado ao processo. Estudos que abordam a fotodegradação de diferentes contaminantes emergentes tem constatado que a degradação, realmente é otimizada com a inserção de semicondutores (Prestes, Giboon, Lanzarin, Moro, 2010; Cruz et al., 2010; Buth, 2009; Hrysyk, 2018; Papamija \& Sarria, 2011; Bernardes et al., 2011), e deste modo, explorar estas alternativas se fazem necessárias.

Deste modo, a aplicação dos semicondutores em processos avançados de oxidação apresentou efetiva ação, na degradação da Sertralina, sendo que a inserção dos agentes dopantes (Mn e $\mathrm{B}$ na proporção de $1 \%(\mathrm{~m} / \mathrm{m})$ quando correlacionado ao Ti) foi capaz de otimizar os processos, uma vez que estes permitem diferentes interações entre o semicondutor e a radiação eletromagnética, tornando o processo mais eficiente.

\section{Conclusões}

A fotocatálise apresenta êxito na degradação da sertralina, em comparativo com a fotólise.O uso de semicondutores dopados tem ação mais eficiente como pode ser observado com $\mathrm{TiO}_{2} 1 \% \mathrm{~B}$, e para o mesmo semicondutor a constante cinética é maior, considerando-se que a degradação da sertralina seja mais eficaz visto que a velocidade de reação é maior.Nesse cenário, percebe-se que há caminhos para a otimização da remoção da sertralina, por meiodo estudo de processos fotocatalíticos aplicáveis para a remoção de compostos persistentes na biota aquática.

\section{Referências}

Bernardes, A. A., Bulhosa, M. C. S., Gonçalves, F. F., D’Oca, M. G. M., Wolke, S. I., Santos \& J. H. Z. (2011). Materiais $\mathrm{SiO} 2-\mathrm{TiO} 2$ para degradação fotocatalítica de Diuron. Química Nova, 34(6), 1343-1348. doi: 10.1590/S0100-40422011000800009.

Buth, D. F. (2009). Degradação fotocatalítica da tetraciclina em solução aquosa empregando TiO2 suportado (Dissertação de Mestrado). Universidade Federal do Rio Grande do Sul, Porto Alegre, Rio Grande do Sul, Brasil.

Cesaro, A. \& Belgiorno, V. (2016). Removal of Endocrine Disruptors from Urban Wastewater by Advanced Oxidation Processes (AOPs): A Review. The Open Biotechnology Journal, 10, 151-172. doi: 10.2174/1874070701610010151

Chen, Y., Li, W., Wang, J., Gan, Y., Liu, L. \& Ju, M. (2016). Microwaveassisted ionic liquid synthesis of Ti3+ self-doped $\mathrm{TiO} 2$ hollow nanocrystals with enhanced visible-light photoactivity. Applied Catalysis B: Environmental, 191(3), 94-105. doi: 10.1016/j.apcatb.2016.03.021

Cruz, L .H., Henning, F.G., Santos, A. B. \& Peralta, Z. P. (2010). Degradação fotocatalítica de sulfametoxazol, trimetoprima e diclofenaco em solução aquosa. Química Nova, 33(6), 1270-1274. doi: 10.1590/S010040422010000600010

Santos, M.C., Oehninger, I.M., Willing, J.C.M. \& Rosa, M.F. (2017). Utilização de fotocatálise heterogênea para a degradação de contaminantes emergentes: cloridrato de norfloxacino. Revista de Química Industrial, 758(1), 25-35.

Hrysyk, A. S. (2018). Degradação fotocatalítica de poluentes emergentes 4 empregando TiO2 imobilizado (Tese Doutorado). Universidade Estadual de Ponta Grossa, Paraná, Brasil.

Junior, I. L. C., Pletsch, A. L. \& Torres, Y. R. (2014). Ocorrência de fármacos antidepressivos no meio ambiente-revisão. Revista Virtual de Química, 6(9), 1408-1431. doi: 10.5935/1984-6835.20140092

Kaplan, R., Erjavec, B., Drazi, G., Grdadolnikc, J. \& Pintar, A. (2015). Simple synthesis of anatase/rutile/brookite $\mathrm{TiO} 2$ nanocomposite with superior mineralization potential for photocatalytic degradation of water pollutants. Applied Catalysis B: Environmental, 181, 465-474. doi: 10.1016/j.apcatb.2015.08.027

Melo, S. A. S., Trovó, A. G., Bautitz, I. R. \& Nogueira, R. F. P. (2009). Degradação de fármacos residuais por processos oxidativos avançados. Química Nova, 32(1), 188-197. doi: 10.1590/S010040422009000100034

Moreira, A. J., Borges, A. C., Gouvea, L. F. C., MacLeod, T. C. O. \& Freschi, G. P. G. (2017). The process of atrazine degradation, its mechanism, and the formation of metabolites using UV and UV/MW photolysis. Journal of Photochemistry and Photobiology A: Chemistry, Vol. 347, pp. 160-167. doi: 10.1016/j.jphotochem.2017.07.022

Moreira, A. J., Pinheiro, B. S., Araujo, A. F. \& Freschi, G. P. G. (2016). Evaluation of atrazine degradation applied to different energy systems. Environmental Science and Pollution Research, Vol. 23, pp. 18502-18511. doi: 10.1007/s11356-016-6831-x

Papamija, M. \& Sarria, V. (2010). Degradación fotocatalítica del ibuprofeno empleando dióxido de titanio. Revista de Ingeniería, 31, 47-53.127.

Peng, Y. P., Loa, S. L., Oua, H. H. \& Lai, S. W. (2010). Microwaveassisted hydrothermal synthesis of N-doped titanate nanotubes for visible-light-responsive photocatalysis. Journal of Hazardous Materials, Vol. 183, pp. 754-758. doi: 10.1016/j.jhazmat.2010.07.090

Perini, P. A. L. \& Nogueira, R. F. P. (2015). Zero-valent iron mediated degradation of sertraline - effect of $\mathrm{H} 2 \mathrm{O} 2$ addition and application to sewage treatment plant effluent. Journal of Chemical Technology \& Biotechnology, Vol. 91, pp. 276-282. doi: 10.1002/jctb.4705

Pliego, G., Xekoukoulotakis, N., Venieri, D., Zazo, J. A., Casas, J. A., Rodriguez, J. J. \& Mantzavinosc, D. (2014). Complete degradation of the persistent anti-depressant sertraline in aqueous solution by solar photo-Fenton oxidation. Journal of Chemical Technology and Biotchnology, 89, 814-818. doi: 0.1002/jctb.4314

Prestes, T. H., Giboon, D. O., Lanzarin, M. A., Moro, C. C. (2010). Degradação fotocatalítica do fungicida Tebuconazole em solução aquosa. Química Nova, 33(4), 798-801. doi: 10.1590/S010040422010000400006

Silva, C. G. A. \& Collins, C. H. (2011). Aplicações de cromatografia líquida de alta eficiência para o estudo de poluentes orgânicos emergentes. Química Nova, 34(2), 665-676. doi: 10.1590/S010040422011000400020

Shen, L. Q., Beach, E. S., Xiang, Y., Tshudy, D. J., Khanina, N., Horwitz, C. P., Bier, M. E. \& Collins, T. J. (2011). Rapid, Biomimetic Degradation in Water of the Persistent Drug Sertraline by TAML Catalysts and Hydrogen Peroxide. Environmental Science and Technology, 45(18), 7882-7887. doi: 10.1021/es201392k

Styrishave, B., Sorensen, B. H. \& Ingerslev, F. (2011). Environmental risk assessment of three selective serotonin reuptake inhibitors int the aquatic environment: a case study including a cocktail scenario. Environmental Toxicology and Chemistry, 30, 254-261. doi: 10.1002/etc.372

Suchara, E. A. (2007). Desenvolvimento de metodologias analíticas para determinação de fármacos em fluidos biológicos e amostras ambientais por cromatografia líquida e gasosa. Tese Doutorado em Química, Centro de Ciências Físicas e Matemáticas/Universidade Federal de Santa Catarina, Florianópolis, Santa Catarina. pp. 145.

\section{Licença Creative Commons CC BY 4.0}

Este artigo foi publicado com acesso aberto para distribuíção sob os termos do Licença de Atribuição Creative Commons, que permite uso irrestrito, distribuição, e reprodução em qualquer meio, desde que o trabalho original seja devidamente citado. 